\title{
COMMENT FAIRE PROGRESSER LA PRATIQUE PEDAGOGIQUE?
}

\author{
Ludmila Ungureanu*, ORCID ID: 0000-0003-4986-9172 \\ Technical University of Moldova, Ștefan cel Mare str. 168,Chișinău, Republic of Moldova \\ *ludmila.ungureanu@lm.utm.md \\ Received: 03.11.2021 \\ Accepted: 04.23.2021
}

\begin{abstract}
This study is the result of a more recent questioning on the limits of the profession of teacher on what we are supposed to practice when we want to build effective approaches to students who have difficulty into university learning. However, periods of didactic crisis are present among experienced teachers. They are justified by the desire to trigger new approaches. This time, it is a question of adopting an innovative pedagogy in the context of the booming development of new technologies, where the role of the teacher is undergoing major changes. This questioning therefore appears following longterm observation and regular consultation within a teaching team in order to advance teaching practice.
\end{abstract}

Keywords: psycho-educational approach, support program, motivational approach, flipped classroom, project approach, problem approach.

Résumé. La présente étude est le fruit d'un questionnement plus récent sur les limites du métier d'enseignant, sur ce qu'on est censé y pratiquer lorsqu'on veut construire des démarches efficaces auprès des étudiants qui ont du mal à rentrer dans les apprentissages universitaires. Or, les périodes de crise didactique sont présentes chez les professeurs chevronnés. Elles se justifient par le désir de déclencher de nouvelles approches. Il est question, cette fois-ci, d'adopter une pédagogie innovante dans le contexte du développement en flèche des nouvelles technologies, où le rôle de l'enseignant subit des modifications importantes. Cette remise en question apparaît donc suite à une observation de longue haleine et une concertation régulière au sein d'une équipe didactique afin de faire progresser la pratique pédagogique.

Mots clé: approche psychopédagogique, programme d'accompagnement, démarche motivationnelle, classe inversée, approche par projet, approche par problème.

Rezumat. Prezentul studiu este produsul unei chestionări mai recente referitoare la limitele meseriei de profesor de limbă franceză pentru străini, la ce ar trebui, acesta din urmă, să recurgă atunci când vrea să elaboreze un demers eficient adresat studenților care îmbrățisează mai anevoios studiile universitare. Or, perioadele de criză didactică nu-i ocolesc nici pe cei cu destulă experiență în domeniu. Ele se justifică prin dorința acestora de a crea noi strategii, fiind vorba, de astă dată, de adoptarea unei pedagogii inovative în 
contextul dezvoltării fulminante a noilor tehnologii, unde rolul profesorului este supus unor modificări importante. Această reconsiderare apare deci ca urmare a unor observări de durată și a unei concertări regulate în sânul unei echipe didactice în vederea eficientizării practicilor pedagice.

Cuvinte cheie: strategie didactică, program de însoțire, demers motivațional, clasă inversată, studiu în bază de proiect, studiu în bază de problemă.

\section{Introduction}

Les jeunes ne veulent plus savoir. C'est le constat qu'on émet le plus souvent dans le domaine de l'éducation. On peut, pourtant, en être fort contrarié, car ceux-ci font preuve de curiosité, même d'une certaine avidité quant à l'information à avaler. Les observations menées auprès des étudiants du premier cycle universitaire nous permettent de constater qu'ils commencent à se désinvestir notamment devant les choses de l'esprit. On atteste, chez eux, l'envie de savoir, d'accéder à la connaissance sans pourtant vouloir apprendre. Savoir, oui; apprendre et penser non. Ils ne veulent pas aller au-delà du voir et de l'entendre pour savoir. Ils ne supportent guère le flottement, le doute, la suspension du jugement qui accompagnent nécessairement toute recherche. Ils vont jusqu'à mettre hors circuit leur fonctionnement mental au moment où il faudra associer, faire des liens, chercher, déduire.

\section{Raisons du désinvestissement intellectuel des apprenants}

Cette image simplificatrice et réductrice du fonctionnement intellectuel de nos apprenants risque d'engendrer le défaitisme pédagogique. Il s'en suit que les enseignants devraient se mettre à la recherche d'une solution viable. Or, il est évident que celle-ci n'est pas possible sans connaître les raisons de ce phénomène comportemental. Pour ce faire il nous a paru utile d'aller chez Boimare (2000), dont le raisonnement sur le phénomène en question présente une approche psychopédagogique [1]. Selon lui, la conduite d'évitement de la pensée porte en elle les germes d'une déstabilisation psychique. En effet, toute recherche, toute entrée dans un code nouveau oblige à prendre des distances avec ce qui est perçu directement, en délaissant certains repères. Ce temps de suspension qui devrait être propice à la construction et à la mise en forme provoque la dispersion et la déroute. Ce vide, cette brèche ne peut pas stimuler l'activité de penser. Face à cette situation, les jeunes cherchent à protéger leur équilibre psychique. Pour cela, ils disposent de trois moyens qu'ils utilisent simultanément : ils coupent le fil de la pensée; ils projettent leur peur et leur inquiétude sur le travail à faire, sur l'enseignant; ils utilisent leur corps comme carapace protectrice. «En effet, le travail intellectuel, la réflexion oblige à se pencher sur son intérieur, son psychisme. Or, le désordre extérieur du jeune n'est que le reflet de son désordre interne qui lui fait peur» [2]. Il s'ensuit que toute question intellectuelle le renvoie à des images internes dangereuses qu'il faut fuir pour ne pas s'exposer au danger.

\section{Démarche psychopédagogique}

En tant qu'enseignants on se demande, en l'occurrence, quoi faire pour s'en sortir? Comment agir pour ne pas éprouver le sentiment d'impuissance et de culpabilité? On comprend que la capacité de didactiser le matériel ne suffit pas. Il faut avoir une certaine disponibilité psychique qui puisse nous aider à ne pas se laisser manipuler ou à ne pas sombrer dans la démagogie ou le laissez-faire. 
Ancrée dans l'approche psychopédagogique sus-évoquée, on dirait avec Boimare que diminuer les causes d'angoisse paraît être l'action prioritaire. Pour ce faire on devrait établir des cadres dans la classe et dans la réflexion. En l'occurrence, une éventuelle structuration de la classe, un certain ordre extérieur va aider à déstructurer le chaos à l'intérieur du jeune.

Le constat que nous venons de faire avec Boimare nous conduit à la nécessité de mettre à jour notre système éducatif fondé sur l'obtention de résultats plutôt que sur le développement des potentiels. Comment faire pour emmener nos apprenants le plus loin possible dans leur apprentissage, ayant la conviction que chaque individu a ses propres ressources? Quelle méthodologie mettre en œuvre pour former des individus autonomes et confiants? Et c'est là que le concept de bienveillance s'impose pour être pleinement analysé. Dans ce contexte on est amené à mettre en cause l'autorité de l'enseignant en tant que tel: il ne doit plus se fonder sur le statut de connaisseur ou détenteur de savoir-faire mais sur sa capacité à susciter la curiosité, à éveiller l'intérêt, à canaliser les efforts vers la réalisation des objectifs ciblés. Et ce qui nous paraît le plus intéressant, attirer les apprenants vers des sujets ou matières qu'il ne maîtrise pas forcément lui-même.

Il s'avère donc important de renouveler notre système éducatif par des programmes d'accompagnement multiples et personnalisés ou apprenants et enseignants apprennent les uns des autres. C'est un schéma vertueux, dont on pourrait s'inspirer dans la création de nos méthodes d'apprentissage.

\section{Le concept de la pédagogie inversée}

Dans le même ordre d'idées on constate que l'internet transforme, à son tour, l'enseignant dans un simple descripteur du monde, une ressource parmi d'autres. Quelles seraient, dans ce nouveau contexte, les innovations pédagogiques les plus abordables?

Il nous paraît intéressant d'y considérer le concept de la «classe inversée» - une innovation pédagogique, constituant dernièrement l'objet d'études de plusieurs chercheurs dont Dumont et Berthiaume, Nizet et Meyer, Tchatouo et Baque, etc. [3 - 5].

Pratiquée surtout aux Etats- Unis, l'innovation en question a réussi à conquérir également les enseignants européens. En effet, l'apprenant regarde la leçon chez lui le soir et le lendemain, le professeur vérifie que la leçon a été assimilée en proposant des exercices d'application, des projets créatifs en équipe. Selon Lebrun (2015), cette forme d'enseignement inverse le travail à distance et le travail en présence: la classe inversée consiste à déplacer la partie magistrale d'un cours à la maison et à utiliser le temps de classe ainsi libéré pour réaliser les devoirs traditionnellement faits à la maison [6]. Cette idée peut être complétée par Dufour (2014) qui précise le caractère des activités à faire à la maison, en autonomie, et en classe: des activités de bas niveau cognitif à la maison et des tâches d'apprentissage de haut niveau cognitif en classe [7].

Il s'avère que la classe inversée arrive à augmenter la volonté d'apprendre, à faire croître l'autonomie chez les apprenants. En plus, ces derniers sont amenés à partager des savoirs et à résoudre des problèmes ensemble. Ce modèle collaboratif que nous sommes en train d'analyser réserve au professeur un nouveau rôle, celui de facilitateur ou guide. Ainsi, on est en train de devenir témoin d'une métamorphose sociétale: d'un monde vertical, hiérarchique où le professeur détenait le savoir absolu à un monde horizontal où les relations se tissent sur le bas, en l'occurrence entre les apprenants eux-mêmes. 
On constate néanmoins que certains auteurs tels que Bissonnette et Gauthier (2012) contestent l'efficacité de cette nouvelle méthode d'enseignement vu le manque de données probantes [8]. Par manque de résultats de recherches rigoureuses sur le rendement scolaire en classe inversée, ces auteurs recommandent une attitude de prudence. Pour autant, nous sommes portée à croire à des témoignages individuels comme preuve d'efficacité de cette méthode innovante et nous nous permettons de faire appel à la pédagogie inversée auprès des étudiants universitaires. En effet, le visionnement en autonomie des séquences vidéo rend plus efficace le travail de compréhension orale organisé en classe. Les apprenants participent plus activement à des activités et manifestent un intérêt soutenu pour le sujet en question. De même, les activités de compréhension des écrits, proposées en classe, sont plus efficaces lorsque les documents d'étude sont lus préalablement à la maison. Cela s'explique par le fait que chaque étudiant prend son temps et travaille à son rythme afin de réaliser les mêmes tâches.

\section{La dynamique motivationnelle}

Une autre question qui préoccupe les chercheurs œuvrant dans le domaine de la psychopédagogie est la motivation de nos apprenants, dont on atteste une chute considérable. Quelles en pourraient être les raisons? Nous nous réservons la liberté de penser que ce sont des facteurs relatifs aux cours suivis par nos étudiants. En effet, la littérature scientifique évoque, dans ce contexte, les activités pédagogiques, les pratiques évaluatives, la relation avec l'enseignant, le climat de la classe. Briand (2016) fait le point sur la question de la dynamique motivationnelle qui anime un étudiant lorsqu'il accomplit une activité pédagogique. Il évoque trois perceptions qu'un étudiant a de l'activité pédagogique qui lui est proposée:

a) la perception qu'il a de la valeur de l'activité: le jugement qu'un étudiant porte sur l'intérêt et l'utilité d'une activité pédagogique en fonction des buts qu'il poursuit;

b) la perception qu'il a de sa compétence: la perception qu'il a de lui-même et par laquelle il évalue sa capacité à accomplir de manière adéquate une activité qu'il n'est pas certain de réussir;

c) la perception qu'il a de la controlabilite: le sentiment de controle qu'il exerce sur le deroulement d'une activite et sur ses consequences [9].

Si ces perceptions sont elevees, l'etudiant sera motive, ce qui aura pour consequence qu'il choisira de s'engager cognitivement dans une activite pedagogique qui lui est proposee et perseverera. Si ces perceptions sont faibles, il sera demotive, ne s'engagera pas dans cette activite et ne perseverera pas.

\section{Les innovations pedagogiques les plus abordables}

En partant de l'idee qu'apprendre est une activite complexe, necessitant un accompagnement humain et technique favorable pour le developpement de la competence, nous nous penchons avec confiance sur la nouvelle conception de l'apprentissage, issue des travaux de la psychologie cognitive et des neurosciences. Cette conception est centree sur l'apprenant et basee sur les théories de l'activité: l'action, la réflexion et la collaboration avec autrui sont les conditions essentielles à l'efficacité de l'apprentissage. Elle s'appuie sur notre fonctionnement biologique, cognitif, affectif et sur notre nature essentiellement sociale. Restant dans cette optique, on peut evoquer les 6 activites pedagogiques proposees par Briand (2016) et generalement pratiquees par les enseignants: approche par projet, etudes de cas, approche par problemes, atelier, seminaire de lecture, expose [10]. Dans nos 
yeux, ce sont des activites susceptibles de stimuler la motivation des apprenants car orientees vers le traitement, la transformation des informations reçues. or, notamment cette transformation merite le nom d'apprentissage en tant que processus cognitif et affectif.

Il nous parait pertinent d'evoquer, egalement, dans ce contexte, les reflexions des enseignants publiees dans la revue Pedagogie collegiale [11], destinee a nous eclairer sur les pratiques novatrices, en alimentant constamment notre reflexion pedagogique: Page M. qui traite de l'apprentissage par probleme, Proulx j., abordant l'approche par projet, Gagnon YCh., qui s'approprie l'etude de cas, etc. [12 - 14].

Les activites pedagogiques sus-enoncees nous paraissent d'autant plus pertinentes qu'elles adoptent la methode inductive, selon laquelle l'experience doit preceder la comprehension: le gout d'apprendre est a ce prix. les methodes inductives veulent partir de la realite, de l'observation et de l'experience pour aller vers l'abstraction.

Il est important de preciser aussi que la capacite d'elaboration et de mise en œuvre des activites pedagogiques innovantes se developpe non seulement suite a des lectures specialisees mais aussi grace a des formations regulieres qui puissent ameliorer, en un temps bref, la conceptualisation et la rentabilisation de celles-ci.

\section{Conclusions}

Ainsi, nous nous sommes préoccupée, dans un premier temps, des empêchements que les apprenants éprouvent face au processus de l'apprentissage et nous avons constaté que ceux-ci sont plutôt d'ordre psychique: la peur face au déséquilibre créé par l'effort intellectuel, d'une part, et le manque de motivation d'autre part.

La réflexion que nous avons développée par la suite nous a amenées vers l'idée qu'il est important de favoriser la dynamique motivationnelle de nos apprenants, en recourant à des activités innovantes inspirées et rendues possibles par le développement des technologies telles que la classe inversée, l'approche par projet, l'étude de cas, l'approche par problèmes, l'atelier, le séminaire de lecture, l'exposé.

Nous avons voulu démontrer que les activités pédagogiques proposées aux étudiants devraient se baser sur deux principes: la diminution de leur état d'angoisse et le maintien de la motivation tout au long de leurs études. Ces activités doivent influer de façon positive sur la valeur que les étudiants leur accordent (perception de l'utilité), sur la perception de leur compétence à les accomplir et sur le sentiment de contrôlabilité de leur déroulement.

\section{Références bibliographiques}

1. Boimare S. L'enfant et la peur d'apprendre, Dunod, 2000.

2. Boimare S. La médiation culturelle au secours des enfants empêchés de penser. 2019.

Récupéré le 06.02.2021 de https://www.bayardeducation.com/nos-supports-pour-lenseignant/sergeboimare-mediation-culturelle-secours-enfants-empeches-de-penser-autres/

3. Dumont A. et Berthiaume, D. La pédagogie inversée: Enseigner autrement dans le supérieur avec la classe inversée. 2016.

Récupéré le 08.02.2021 de https://biblio.helmo.be/opac_css/doc_num.php?explnum_id=4010

4. Nizet I. et Meyer, F. La classe inversée : que peut-elle apporter aux enseignants ? 2015.

Récupéré le 03.03.2021 de https://www.reseau-canope.fr/agence-des-usages/la-classe-inversee-que-peutelle-apporter-aux-enseignants.html

5. Tchatouo L.P.N., Baque, N. Pédagogie de la classe inversée : place des outils et ressources numériques dans cette forme d'enseignement. 2017. Récupéré le 03.03.2021 de http://www.adjectif.net/spip/spip.php?article427

6. Lebrun M. L'hybridation dans l'enseignement supérieur : vers une nouvelle culture de l'évaluation? Evaluer. 2015. Récupéré le 19.03.2021de: https://www.researchgate.net/publication/272503639_ L'hybridation_dans_l'enseignement_superieur_vers_une_nouvelle_culture_de_l'evaluation 
7. Dufour H. La Classe Inversée. 2014. Récupéré le 11.02 .21 de: http://eduscol.education.fr /sti/sites/eduscol.education.fr.sti/files/ressources/ techniques/6508/6508-193-p44.pdf.

8. Bissonnette, S. et Gauthier, C. Faire la classe à l'endroit ou à l'envers ? Formation et profession. 2012. Récupéré le 08.02.2021 de http://formationprofession.com/files/numeros/1/v20_n01_173.pdf

9. Briand M. La motivation des etudiants a l'universite: mieux comprendre pour mieux agir. 2016. recupere le 17.02.2021 de https://www.innovation-pedagogique.fr/article387.html

10. Pédagogie collégiale :

Récupéré le 11.03.2021 de https://eduq.info/xmlui/handle/11515/36?locale-attribute=en

11. Pagé M. Quand un problème devient fantastique. Compte rendu d'une expérimentation de l'apprentissage par problèmes dans un cours de littérature. Pédagogie collégiale, 2014, vol.18, nr. 1. Récupéré le 02.03.2021 de https://cdc.qc.ca/ped_coll/pdf/Page_18_1.pdf

12. Proulx J. L'apprentissage par projet. Presses de l'Université du Québec, 2008.

13. Gagnon Y. Ch. L'étude de cas comme méthode de recherche. Presses de l'Université du Québec, 2005.

14. Viau R., Joly J., Bédard D. La motivation des étudiants en formation des maîtres à l'égard d'activités pédagogiques innovatrices. Revue des Sciences de l'Éducation, 30 (1), 2004.

Récupéré le 6.02.21 de https://www.erudit.org/fr/revues/rse/2004-v30-n1-rse962/011775ar.pdf 